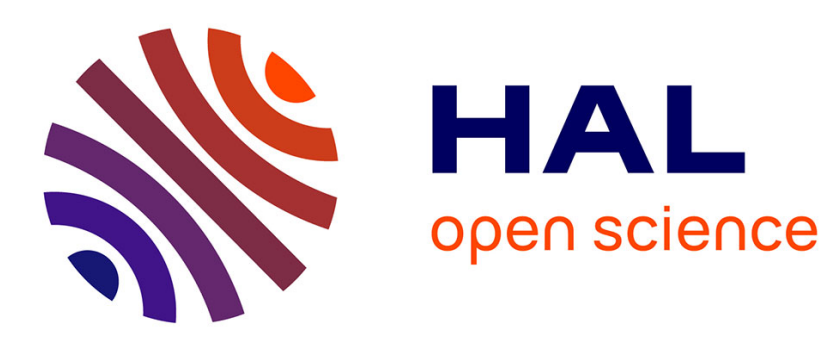

\title{
Characterization and modeling of electrical transport in undoped hydrogenated microcrystalline silicon
}

\author{
P. Abboud, F. Martinez, R. Amrani, D. Habib, S. Parola, Y. Cuminal
}

\section{To cite this version:}

P. Abboud, F. Martinez, R. Amrani, D. Habib, S. Parola, et al.. Characterization and modeling of electrical transport in undoped hydrogenated microcrystalline silicon. Journal of Non-Crystalline Solids, 2017, 477, pp.42 - 49. 10.1016/j.jnoncrysol.2017.09.045 . hal-01654998

\author{
HAL Id: hal-01654998 \\ https://hal.science/hal-01654998
}

Submitted on 7 Jan 2021

HAL is a multi-disciplinary open access archive for the deposit and dissemination of scientific research documents, whether they are published or not. The documents may come from teaching and research institutions in France or abroad, or from public or private research centers.
L'archive ouverte pluridisciplinaire HAL, est destinée au dépôt et à la diffusion de documents scientifiques de niveau recherche, publiés ou non, émanant des établissements d'enseignement et de recherche français ou étrangers, des laboratoires publics ou privés. 


\section{Characterization and Modeling of Electrical}

\section{Transport in Undoped Hydrogenated}

\section{Microcrystalline Silicon}

\section{DOI : 10.1016/j.jinoncrysol.2017.09.045}

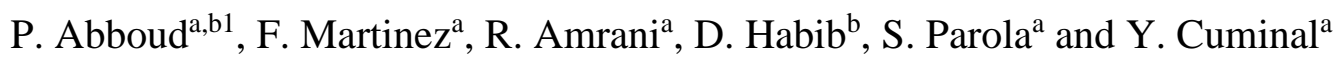

${ }^{a}$ Institute of Electronics and Systems (IES), University of Montpellier, UMR CNRS 5214, 860 Rue St-Priest, 34090 Montpellier, France.

b Applied Physics Laboratory, Lebanese University, Faculty of Science II, campus Fanar, 90656 Lebanon.

\section{Abstract}

In this paper, we present the characterization and modeling of the electrical transport of hydrogenated microcrystalline silicon $(\mu \mathrm{c}-\mathrm{Si}: \mathrm{H})$. Electrical conductivity measurements for several crystalline fractions have been carried out. The latter have been combined with surface potential measurements from atomic force microscopy in order to investigate the electrical transport in the heterogeneous structure. We propose a new numerical model based on a three-dimensional electrical circuit to extract the parameters involved in the transport. A physical based model of tunneling at large grain boundary is implemented. Combining the latter with other conductivities in the large electrical circuit, the percolation behavior in respect to crystalline fraction is fully simulated. A benefit of such a model is that it captures the fundamental physics phenomena with only a few comprehensible parameters.

Index Terms: microcrystalline silicon, percolation, transport, AFM.

\footnotetext{
${ }^{1}$ Corresponding author

E-mail: pascale.abboud@live.com, present address: EC2M, Faculty of Science 2, Campus Pierre Gemayel, Fanar, Lebanese University,90656 Lebanon. 


\section{Introduction}

Hydrogenated microcrystalline silicon $(\mu \mathrm{c}-\mathrm{Si}: \mathrm{H})$ is a promising material to improve the performance of transistors [1] and photovoltaic devices [2] based on hydrogenated amorphous silicon (a-Si:H). Since $\mu \mathrm{c}-\mathrm{Si}: \mathrm{H}$ can be prepared at low temperatures using equipment similar to that used for a-Si: $\mathrm{H}$, this material is positioned as a potential candidate for low cost applications. Many publications have reported improvement in conductivity with the increase in the crystalline volume fraction [3,4]. This behavior is related to the creation of percolation conduction paths through the silicon crystallites embedded in the a-Si:H.

Temperature dependence of the dark conductivity has been investigated mainly for high crystallinity samples. For such a crystalline fraction, Konezny has considered the film to be homogeneous and the transport is modeled by an array of tunnel junctions at the region of closest approach between the crystallites [5]. The percolation behavior of this mixed-phase compound cannot be taken into account and renders questionable the physical meaning of the extracted parameters.

Many authors have reported the percolation behavior of the $\mu \mathrm{c}-\mathrm{Si}: \mathrm{H}$ and the effective medium approximation (EMA) has been used to investigate the electrical properties of the film $[3,4,6,7]$. The main objective is to predict the global conductivity of a random mixture of particles of two different conductivities. The EMA can randomly describe an inhomogeneous medium with various sizes of spherical or ellipsoidal inclusions. The EMA correctly predicts the percolation phenomena for high crystalline volume fractions. However, the prediction of the percolation threshold strongly depends on the choice of EMA theory e. g. Bruggeman theory, Maxwell Garnett or Wood-Ashcroft mixing rule. All these models predict a percolation threshold from $30 \%$ to $70 \%$ [4]. Due to the wide range of predicted thresholds, the EMA appears to be inaccurate when investigating the complex structure of the $\mu \mathrm{c}-\mathrm{Si}: \mathrm{H}$, especially for crystalline volume fractions around the percolation threshold.

A three-dimensional (3D) model has been proposed by Overhof [8]. Based on a conductance network calculation, the authors simulate the percolation behavior for the conductivity and Hall mobility with a percolation threshold around 30\%. However, the material, modeled as a homogenous random mixture of two phases, is far from the actual microstructure of the $\mu \mathrm{c}-\mathrm{Si}: \mathrm{H}$ and the microscopic behavior of the transport (especially tunneling at grain boundary) is not included in this model.

In this paper, we present a study of the electrical properties of $\mu \mathrm{c}-\mathrm{Si}: \mathrm{H}$ films. Atomic force microscopy (AFM) measurements and electrical characterizations have been carried out for 
several crystalline volume fractions. Based on this experimental data, we are able to present a numerical 3D model of the electrical transport. The main feature of this model is to unify the different approaches and provide a comprehensive tool capable of investigating the microscopic and macroscopic behavior of $\mu \mathrm{c}-\mathrm{Si}: \mathrm{H}$.

In order to obtain realistic simulations and to eliminate the limitations of the Overhof's model, we have implemented the 3D discrete dynamical growth model of the $\mu \mathrm{c}-\mathrm{Si}: \mathrm{H}$ originally proposed by Bailat [9]. This model provides a 3D description of the film, including transition from the amorphous to the crystalline phase, conical shape of the crystalline domains, crystalline fraction and roughness evolution with respect to the layer thickness.

The numerical model of transport deals with a very large conductance network, taking into account the transport in amorphous and crystalline phases and also transitions at grain boundary so that we are able to obtain both microscopic understanding of the transport phenomenon as well as macroscopic percolation behavior of the film.

\section{Experimental details}

Argon diluted a-Si:H and $\mu \mathrm{c}-\mathrm{Si}: \mathrm{H}$ thin films were deposited in a conventional RF (13.56 $\mathrm{MHz}$ ) PECVD chamber at a substrate temperature of $200^{\circ} \mathrm{C}$. Various crystalline volume fractions were achieved by modifying the gas pressure and RF power [10,11]. The crystallinity was characterized by Raman scattering measurements with an Argon laser at a wavelength of $473 \mathrm{~nm}$. Surface native oxide was removed by HF etching followed by evaporation through a photolithographic mask with a $300 \mathrm{~nm}$ thickness of aluminum contacts having a width of $200 \mu \mathrm{m}$ and a length of $2500 \mu \mathrm{m}$. The conductivity measurements were carried out in dark and vacuum in a coplanar configuration. Before the measurements, all samples were heated at $425 \mathrm{~K}$ for 30 minutes to remove the impurities present on the surface. The conductivity has been measured using an Agilent 4156C semiconductor parameter analyzer.

\section{Electrical conductivity measurements}

Fig.1 shows typical examples of the variation of conductivity versus temperature for the samples of crystalline fractions $0 \%, 21 \%, 54 \%, 61 \%$ and $70 \%$. The sensitivity of the experimental set-up reduces the temperature range studied according to the conductivity of each sample. It goes down to $285 \mathrm{~K}$ for the more amorphous and to $175 \mathrm{~K}$ for the more crystalline samples. Over the range studied, a thermally activated conductivity is observed, 
from which we can extract the activation energy $\left(E_{a}\right)$ and conductivity prefactor $\left(\sigma_{0}\right)$ according to the Arrhenius equation:

$$
\sigma=\sigma_{0} \exp \left(-\frac{E_{a}}{K_{B} T}\right)
$$

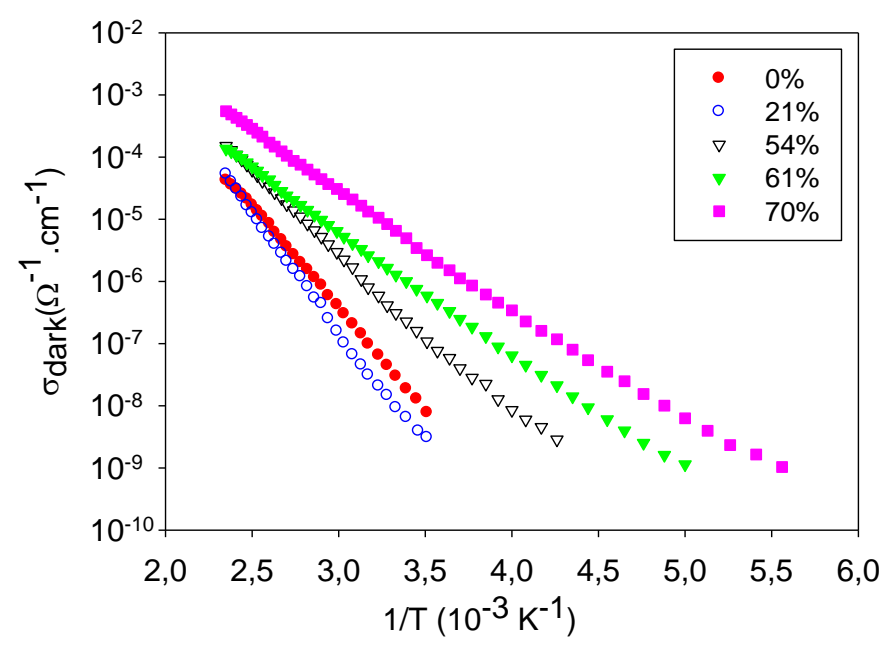

Fig. 1. Examples of Arrhenius plot of the dark conductivity for different crystalline fractions.

We present in Fig.2 the dependence of $E_{a}$ and $\sigma_{0}$ on the crystalline fraction. The variations of $E_{a}$ are correlated to the structure of the material. Below the percolation threshold, $E_{a}$ has an average value of $0.75 \mathrm{eV}$, above the percolation threshold this value is $0.37 \mathrm{eV}$.

This sharp drop in $E_{a}$ and $\sigma_{0}$ is related to the change of the microstructure and the formation of percolation paths through a network of crystalline grains. 


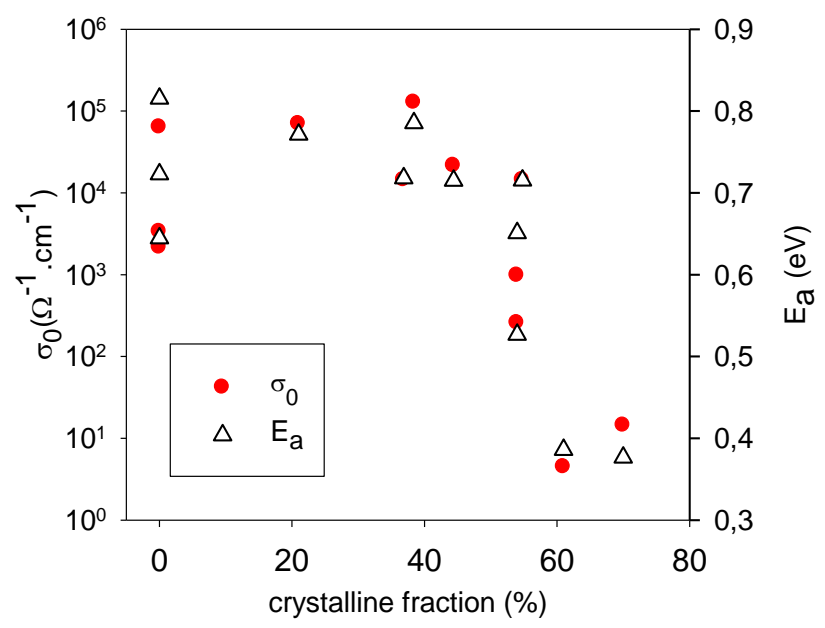

Fig. 2. Variation of the activation energy (triangle) and the conductivity prefactor (closed circle) as a function of the crystallinity.

These experimental results are in agreement with most published data relative to undoped $\mu \mathrm{c}-\mathrm{Si}: \mathrm{H}[12-15]$.

\section{AFM characterizations}

In order to investigate the microscopic properties of the samples, characterizations of the morphology and the surface potential of the samples have been carried out with an atomic force microscope Nanoscope Multimode from VEECO Instruments. The scans were carried out using a platinum coated silicon tip in $2 \times 2 \mu \mathrm{m}^{2}$ area.

Fig. 3 shows the surface morphology in tapping mode for the sample of crystalline fraction $60 \%$. The scan reveals crystalline conglomerates (large grains) and encapsulated crystallites within them. The root mean square (RMS) surface roughness and average large grain diameter were determined using image analysis software "Guiddyon". Results are shown in Fig. 4. 


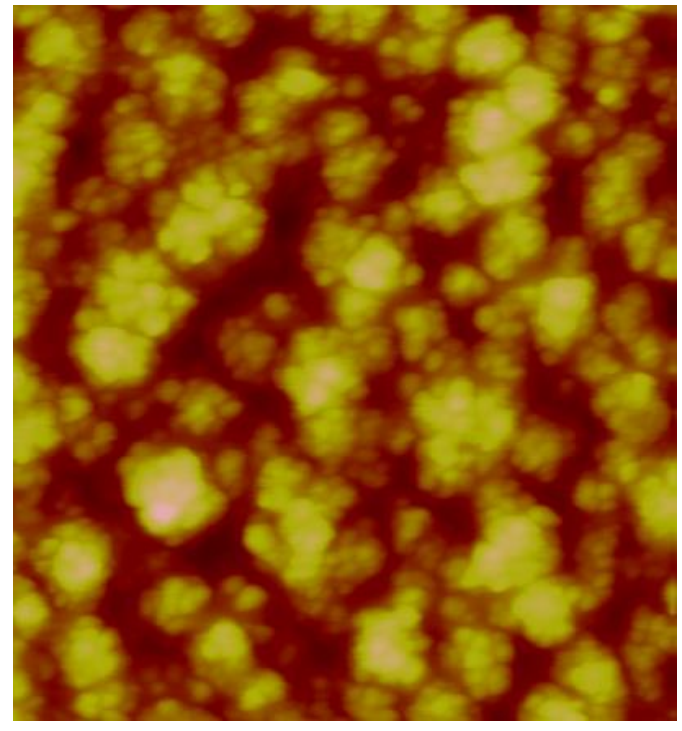

heiaht

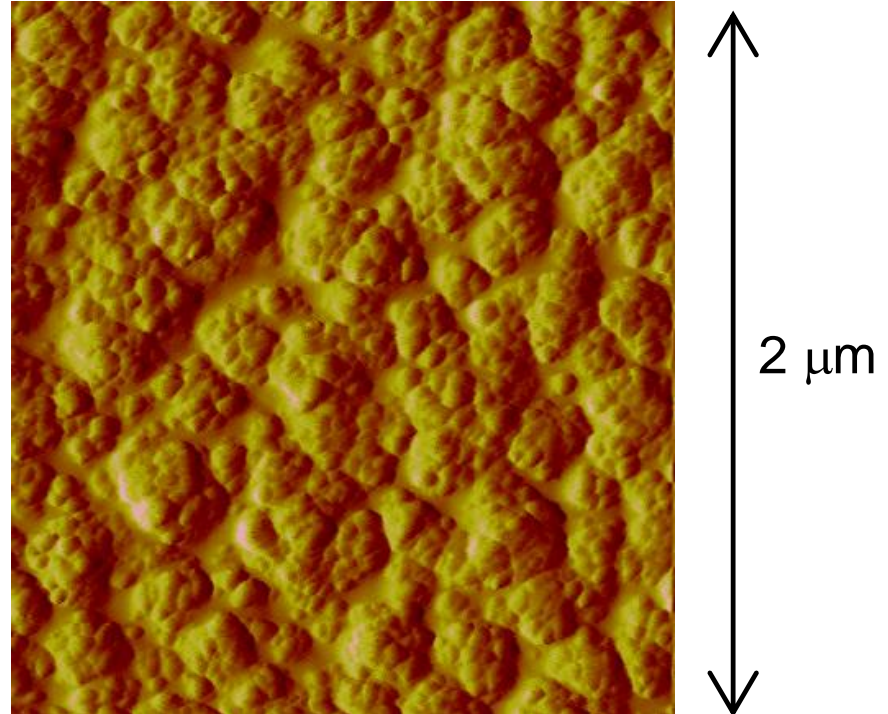

phase

Fig. 3. AFM topographic (left) and phase (right) images on the sample of crystalline fraction $60 \%$.

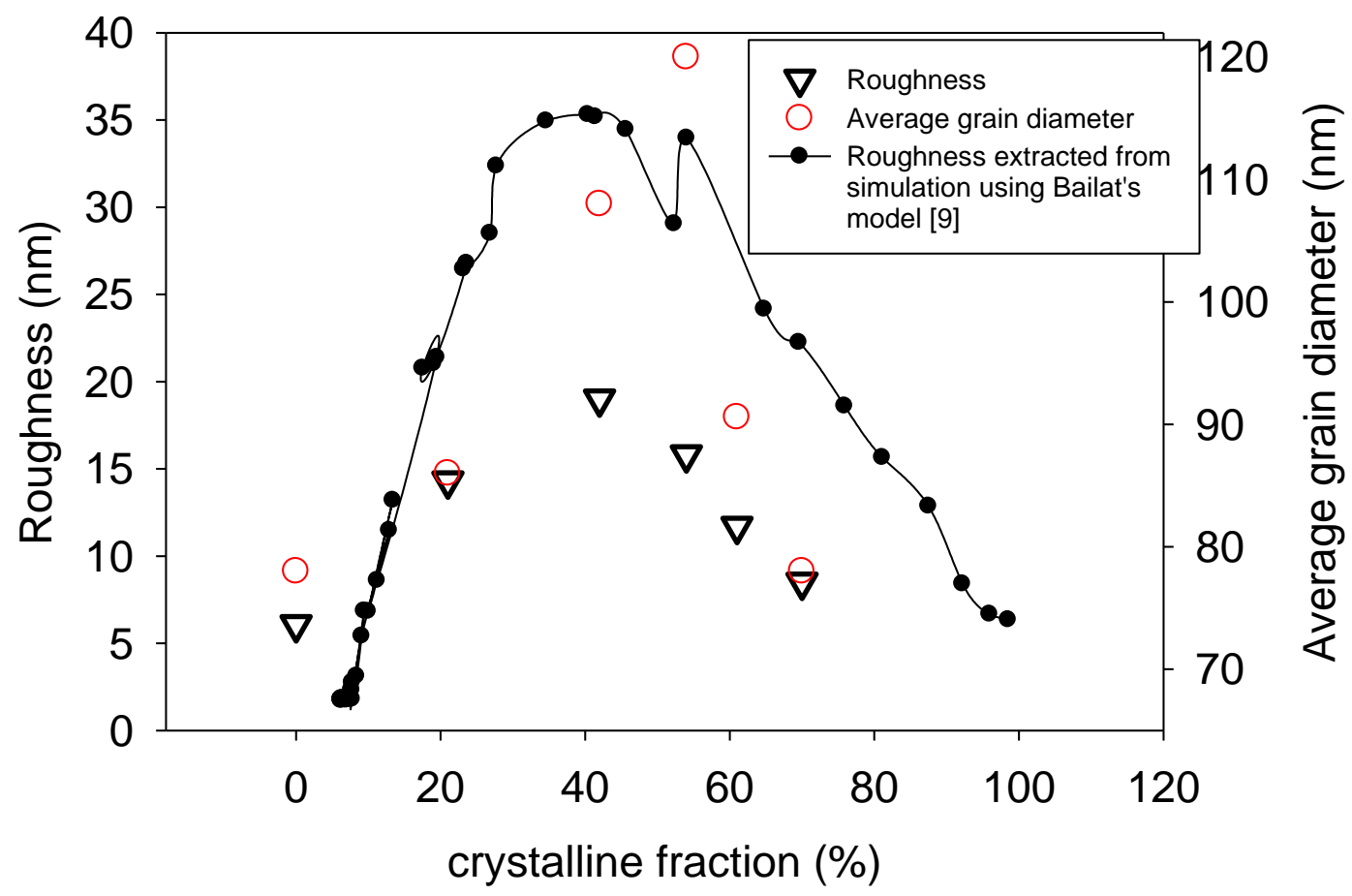

Fig. 4. Experimental RMS roughness (triangles) and average grain diameter (open circles) estimated from AFM measurements, and simulated roughness (line/closed circles) extracted from the simulated structures. 
Surface potential measurements were carried out on two samples of crystalline fractions of $21 \%$ and $54 \%$. A constant voltage is applied between two aluminum coplanar contacts spaced $50 \mu \mathrm{m}$ apart. The AFM tip measures the surface potential of an area of $2 \mu \mathrm{m}^{2}$ located between the contacts. Fig. 5 and Fig. 6 show the morphology and the corresponding surface potential of these two samples.
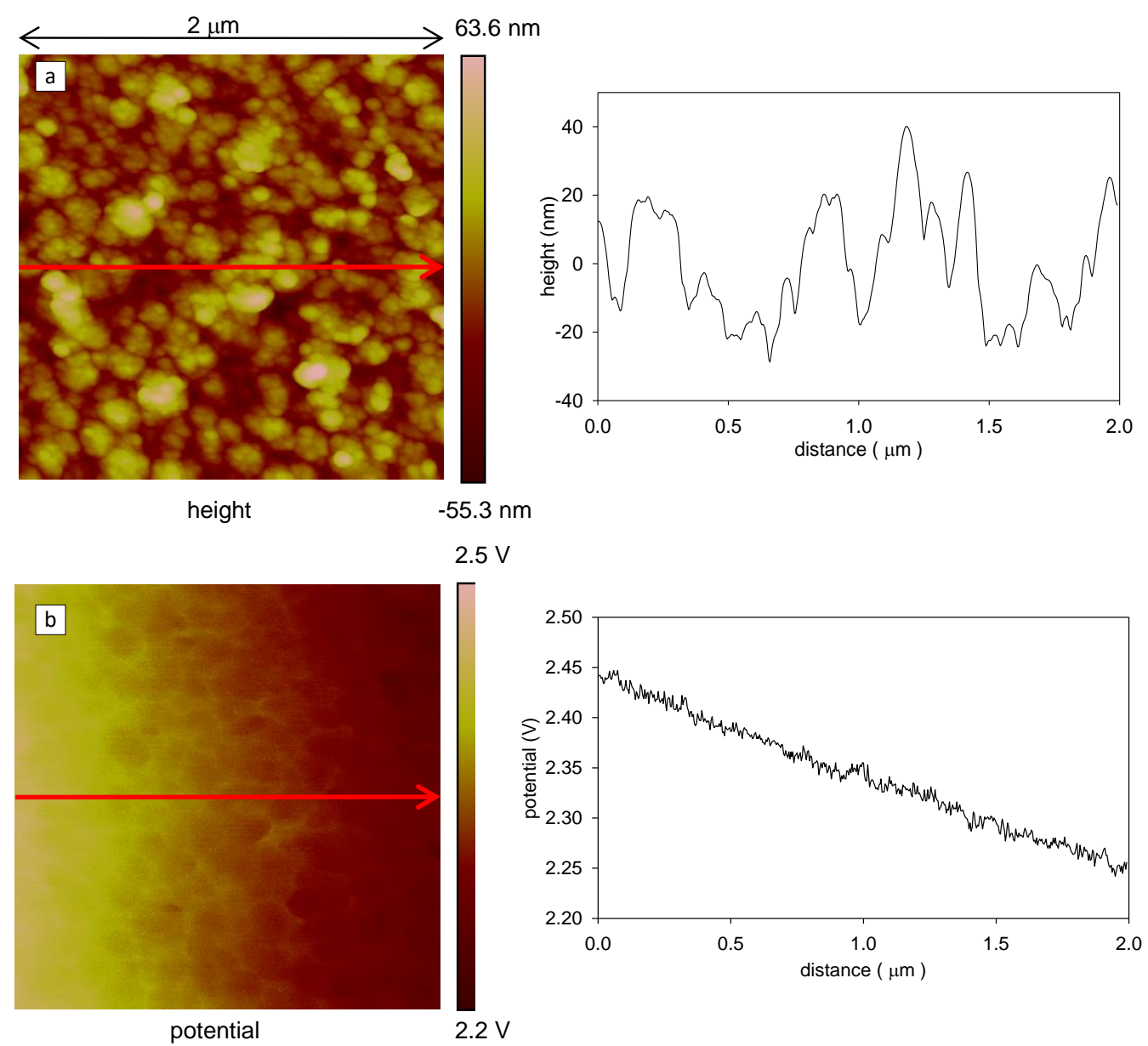

Fig. 5. (a) Surface morphology of the sample of crystallinity $21 \%$, the right side graph shows the variations of the surface height along the arrow. (b) Electrostatic potential measured on an area of $2 \mu \mathrm{m}^{2}$ between two coplanar contacts submitted to a $6 \mathrm{~V}$ bias, the right side graph shows the variations of the potential along the arrow. 


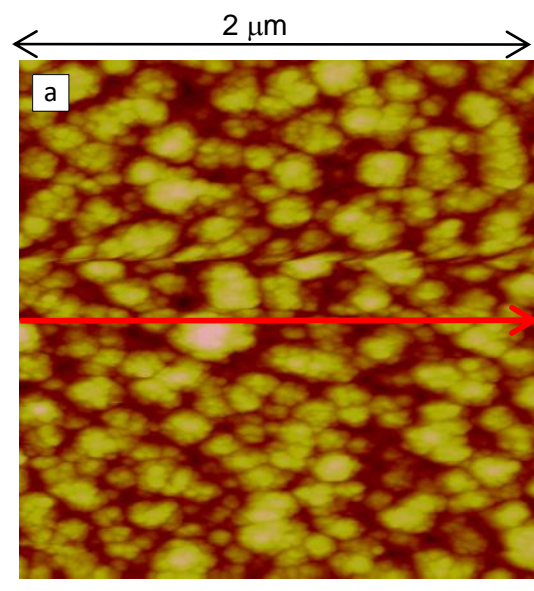

$46.5 \mathrm{~nm}$

height

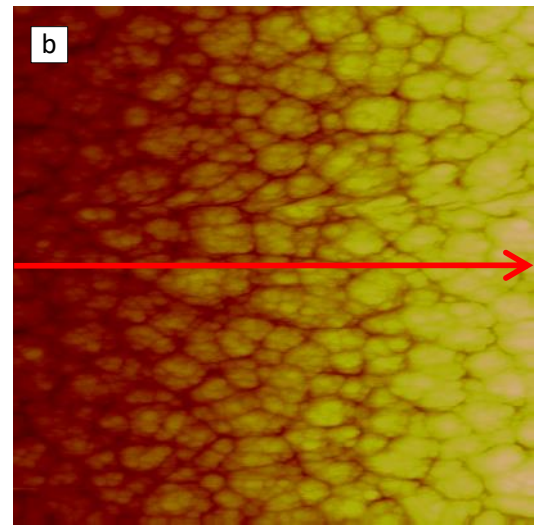

potential

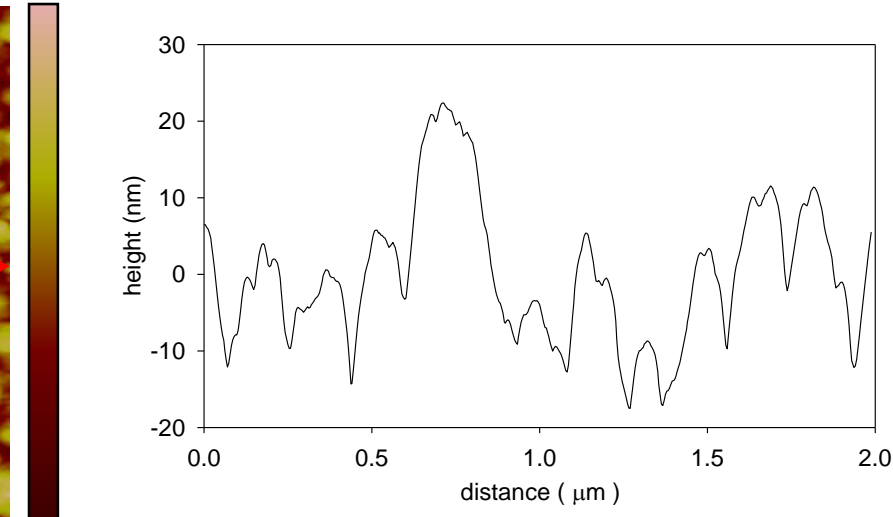

$-45.7 \mathrm{~nm}$

$3.6 \mathrm{~V}$

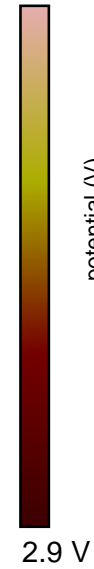

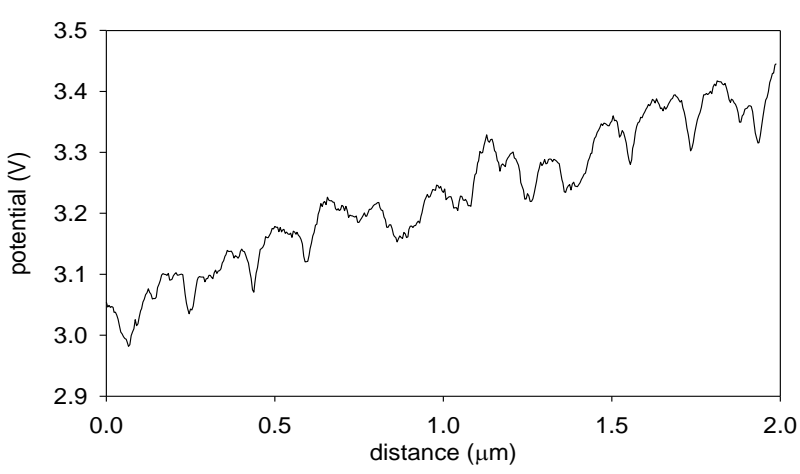

$.9 \mathrm{~V}$

Fig. 6. (a) Surface morphology of the sample of crystallinity $54 \%$, the right side graph shows the variations of the surface height along the arrow. (b) Electrostatic potential measured on a surface of $2 \mu \mathrm{m}^{2}$ between two coplanar contacts submitted to a $12 \mathrm{~V}$ bias, the right side graph shows the variations of the potential along the arrow.

The potential in the sample of crystallinity $21 \%$ is almost linear (Fig. 5 (b)) without any correlation to the surface topography. This result suggests that the transport takes place mainly in the homogeneous amorphous phase of this sample.

The potential in the sample of crystallinity 54\% (Fig.6 (b)) shows a correlation with the topography: potential drops are located at the large grain boundaries.

The surface potential measurements provide a fundamental result to identify current routes in this material. This experimental data suggests that the transport mainly takes place through the large grain boundaries where a potential drop is measured. The quasi constant potential inside the grains suggests that the conductivity inside the grains is high compared to that of 
the boundary, so that the conductivity inside the large grain has a minor influence and represents no barrier for transport inside a large grain, as reported in [16,17].

Moreover, these observations are consistent with many published models where the transport in highly crystalline materials is dominated by the potential barriers at the grain boundaries $[5,13,14,18]$.

In the next section, we present a numerical model based on these experimental results, including macroscopic behavior (percolation) and microscopic behavior (tunneling at grain boundary) in order to extract physical meaning parameters of the electrical transport in this mixed-phase compound.

\section{The model}

\subsection{Modeling of material growth}

In order to obtain a realistic structural model of the material, we have implemented a threedimensional model of growth of microcrystalline silicon based on the work of Bailat [9]. This model involves the fundamental growth mechanisms that determine the microstructure of $\mu \mathrm{c}$ $\mathrm{Si}: \mathrm{H}$, i.e. random deposition of particles, local relaxation and desorption. These mechanisms have been introduced using a set of simulation parameters (n, t, d).

The elementary particle in this model is represented by a cube and depicts the average behavior of a large number of atoms. A cube is released from a random position above the surface and follows a vertical trajectory to attain the surface. The cube then moves to the lowest empty site within its nearest neighbors to smooth the growth surface.

After being incorporated into the material, the cube takes a particular state among " $n$ ". This parameter indicates the maximum number of crystalline states present in the structure. For this purpose, the most represented state among the 25 nearest neighbors of the cube is evaluated: 9 sites below, 8 sites around and 8 sites above. If the number of the cubes having this dominant state is greater than the parameter " $t$ ", the newly deposited cube is given this state. Otherwise, it is given a random state among " $n$ ". " $t$ " is then the minimum number of cubes forming a crystalline germ of the same state, where the growth of a large crystalline grain begins.

The parameter " $\mathrm{d}$ " represents the probability of desorption phenomenon. This parameter describes the preferential etching of the disordered material and is related to the deposition conditions. The newly deposited cube is removed or bonded according to a probability law 
given by the ratio of disorder in its neighborhood. More details about the simulation parameters (n, t, d) and their influence on the structure can be found in the work of Bailat [9].
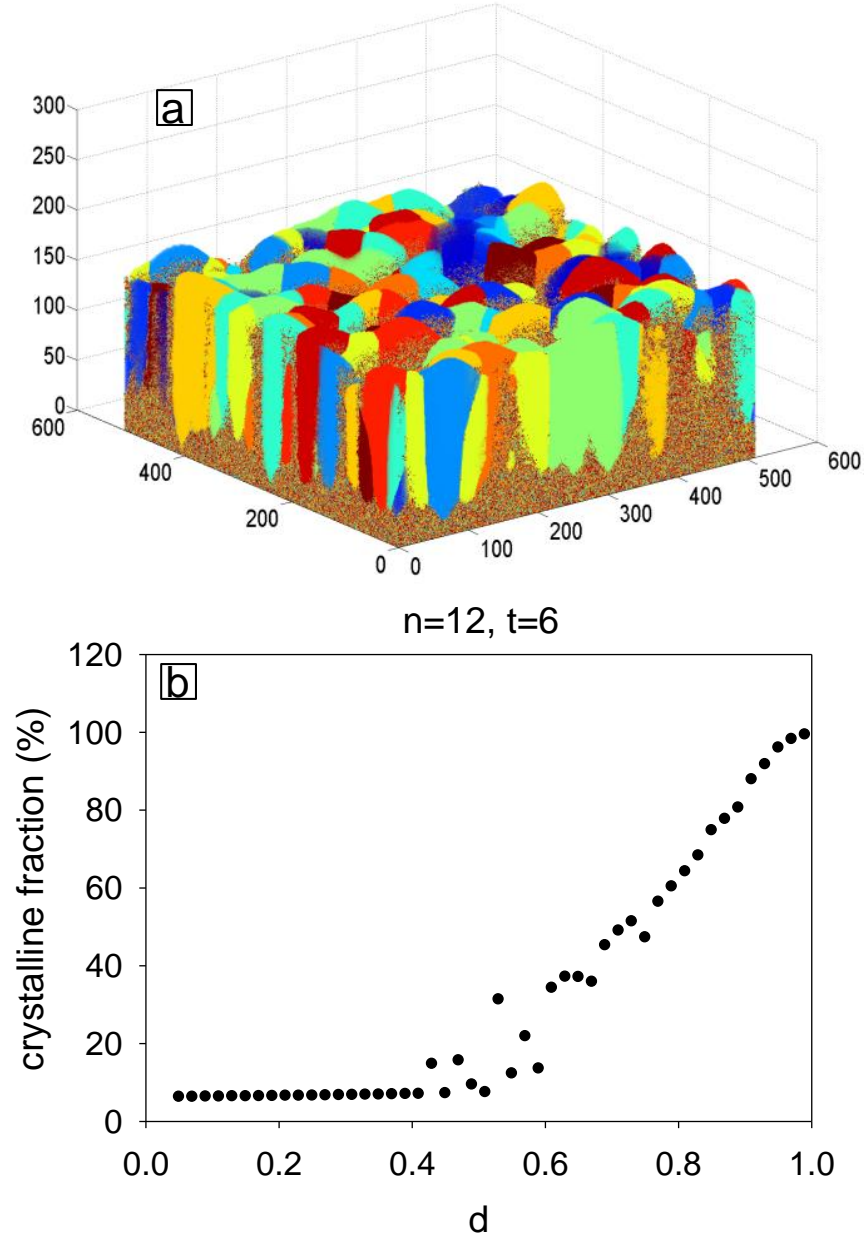

Fig.7. (a) Example of simulated $\mu \mathrm{c}-\mathrm{Si}: \mathrm{H}$ with $\mathrm{n}=12, \mathrm{t}=6$ and $\mathrm{d}=0.8$. The simulation is carried out in $512 \times 512 \times 256$ network units. The uniform domain represent the microcrystalline grains and the dotty domain represent the amorphous phase.(b) Evolution of the crystalline fraction with respect to the desorption process probability $\mathrm{d}$ with $\mathrm{n}=12, \mathrm{t}=6$.

The simulations were carried out in $512 \times 512 \times 256$ network units. Each state is defined by a color, and the main features of the growth dynamics and the complex microstructure of $\mu \mathrm{c}-$ $\mathrm{Si}: \mathrm{H}$ are reproduced: conical shape of crystalline grains, amorphous to crystalline transition and surface roughness evolution.

Fig.7 (a) shows a simulated structure using the parameters: $n=12, t=6$ and $d=0.8$. The crystalline region is defined by the domain where the cubes have the same color. In contrast, the amorphous region is filled with randomly varying colors of cubes. The structure exhibits a mixed-phase material: conical crystalline columns in an amorphous matrix. 
This model has been calibrated by comparing the AFM measurements (roughness, grain size) of the samples to the simulation results. Fig. 4 presents the simulated roughness compared to experimental data from the AFM. We have obtained the best agreement with $\mathrm{n}=12, \mathrm{t}=6, \mathrm{u}=3 \mathrm{~nm}$ whereby " $\mathrm{u}$ " is the size of the network unit and " $\mathrm{d}$ " is varied from 0 to 0.9 . As presented in Fig. 7 (b), the crystallinity depends essentially on the parameter "d".

Finally, the $512 \times 512 \times 256$ network is used as a discrete mesh of the compound for the electrical transport model.

\subsection{Electrical model}

The main challenge in this model is to combine the macroscopic and microscopic effects. At the grain boundary scale, the numerical simulation of a tunnel junction requires a mesh in the order of nanometers or less. On the other hand, it is mandatory to simulate a structure in the micrometer range in order to take into account the macroscopic effects such as percolation and dispersion.

To address this multi-scale modeling problem, we propose to gather the microscopic phenomena (i.e. tunneling) in an electrical circuit comprising a large number of electrical nodes.

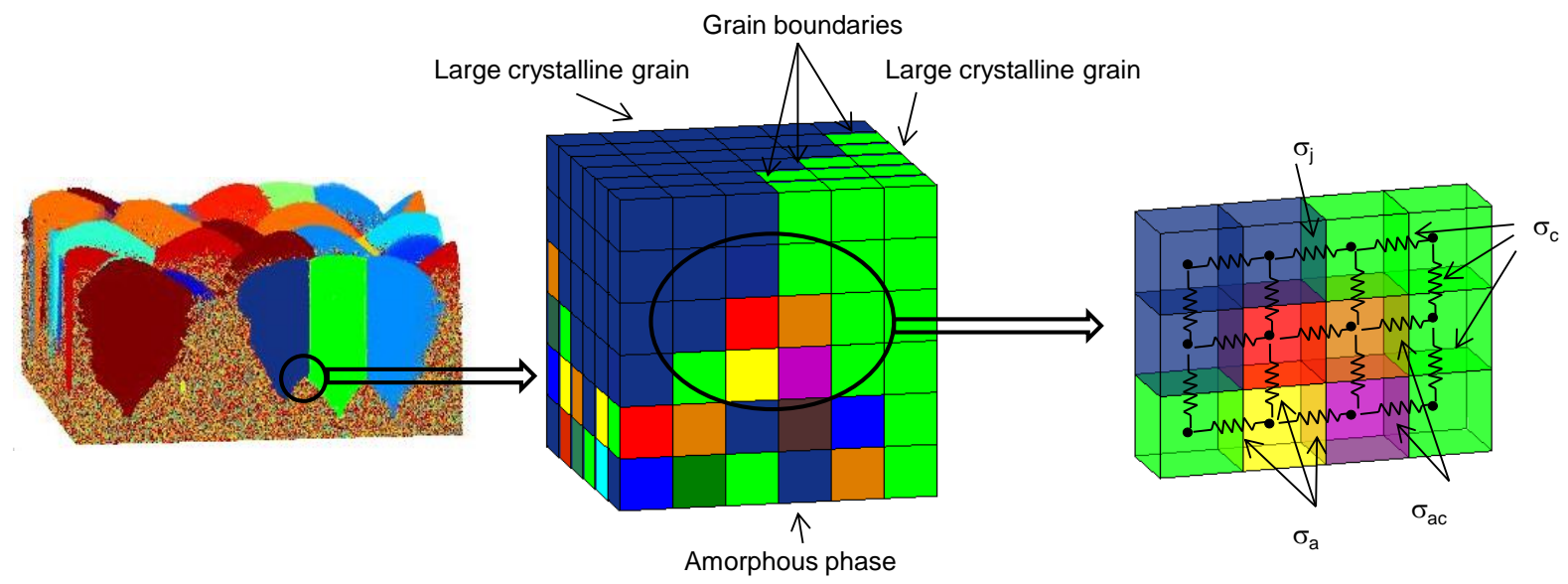

Fig. 8. From the growth material simulation, an equivalent electrical circuit is built taking into account of the nature of the cubes. A set of identical state cubes represents a large crystalline grain, whereas others represent the amorphous matrix.

The approach with a large equivalent electrical circuit has already been used successfully by our team for the modeling of carbon nanotube networks [19]. Considering the large matrix size resulting from the discretization $\left(>10^{6}\right)$, this new solver represents a major evolution of the code developed previously. 
The growth simulation provides a structure consisting of elementary cubes each with a crystalline orientation. A set of identical state cubes represents a large crystalline grain, whereas others represent the amorphous matrix. We have considered each cube as an electrical node of the equivalent circuit. The transport between the cubes is modeled by different dipoles. Fig. 8 shows an example of the equivalent electrical circuit compiled depending on the state of each cube and its nearest neighbors.

$\sigma_{c}$ : the conductivity between two crystalline cubes inside a large crystalline grain.

$\sigma_{\mathrm{j}}$ : the conductivity between large crystalline grains. It depicts the potential barrier at the boundary between two large grains.

$\sigma_{a}$ : The conductivity between two cubes considered as amorphous. It depicts the amorphous phase in $\mu \mathrm{c}-\mathrm{Si}: \mathrm{H}$.

$\sigma_{\mathrm{ac}}$ : The conductivity between an amorphous cube and a crystalline cube. It depicts the potential barrier of the heterojunction c-Si/a-Si.

\section{Results and discussion}

\subsection{Parameters extraction}

In order to compare the experimental results with those simulated, we must take into consideration the crystalline fraction measured by Raman spectroscopy. Using a laser wavelength of $473 \mathrm{~nm}$, the Raman spectroscopy explores the first hundredths of the thickness of the samples [20]. The crystalline fraction in the numerical model is calculated over $200 \mathrm{~nm}$ thickness.

Fig. 9 presents the experimental and simulated conductance normalized to the length/width ratio of the contacts at $300 \mathrm{~K}$. Below the percolation threshold, the conductance is between $10^{-13}$ and $10^{-12} \Omega^{-1}$. At $55 \%$, it increases to attain $10^{-9} \Omega^{-1}$ at crystalline fraction of $70 \%$. A good match is found with a percolation threshold of 55\% using the parameters in table 1.

\begin{tabular}{rcc}
\hline $\begin{array}{c}\sigma_{\mathrm{a}} \\
\left(\Omega^{-1} \cdot \mathbf{c m}^{-1}\right)\end{array}$ & $\sigma_{\mathrm{c}}\left(\Omega^{-1} \cdot \mathbf{c m}^{-1}\right)$ & $\sigma_{\mathrm{j}}\left(\Omega^{-1} \cdot \mathbf{c m}^{-1}\right)$ \\
\hline $3 \times 10^{-8}$ & $4.5 \times 10^{-3}$ & $2 \times 10^{-5}$ \\
\hline
\end{tabular}

Table 1 : adjusted electrical parameters at $300 \mathrm{~K}$. 


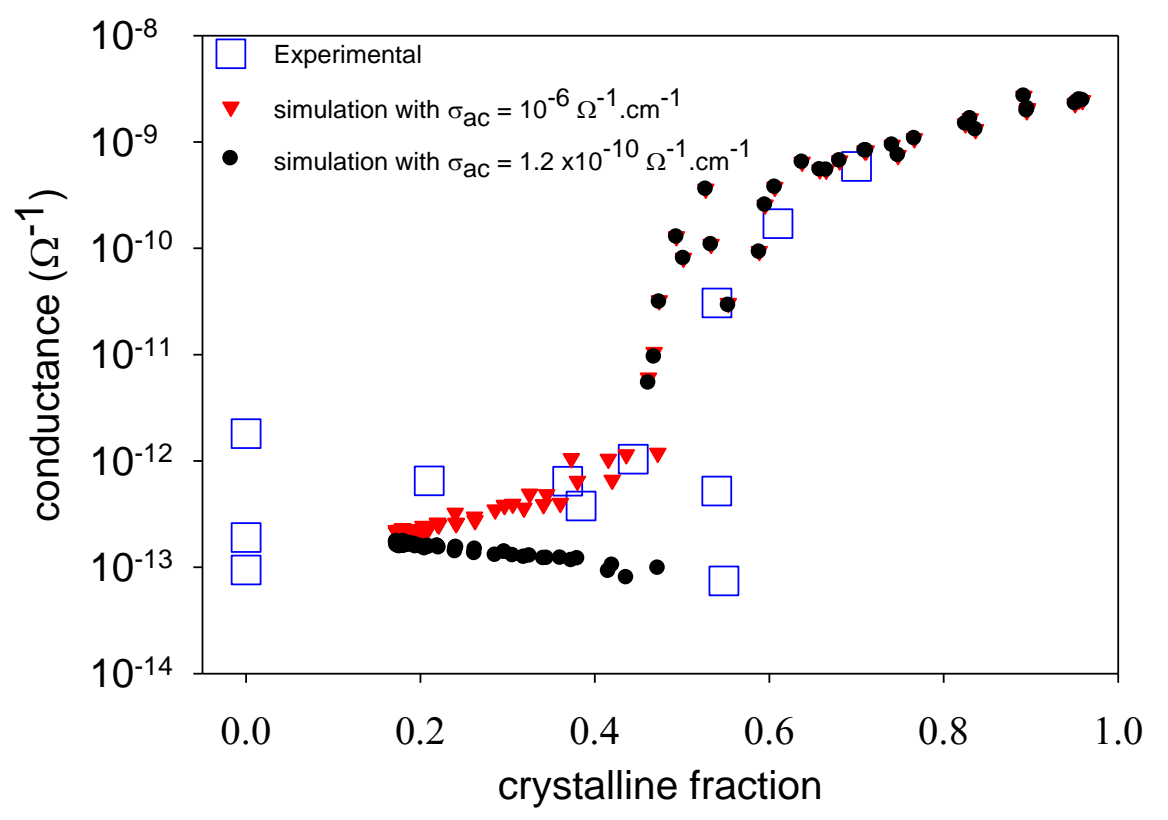

Fig. 9. Experimental (open squares) and simulated normalized conductance (solid triangles with $\sigma_{\mathrm{ac}}=10^{-6} \Omega^{-1} \cdot \mathrm{cm}^{-1}$ and solid circles with $\sigma_{\mathrm{ac}}=1.2 \times 10^{-10} \Omega^{-1} \cdot \mathrm{cm}^{-1}$ ) versus crystalline fraction. Fitting parameters are given in Table 1, except $\sigma_{\mathrm{ac}}$ which is given in the legend and showing the impact on the curve around the percolation threshold.

$\sigma_{\mathrm{c}}$ and $\sigma_{\mathrm{j}}$ have a major influence on the transport in the crystalline phase, while $\sigma_{\mathrm{a}}$ has a direct influence on the values of conductivity below the percolation threshold. The electrical parameters obtained are consistent with usual values of conductivity of amorphous silicon. $\sigma_{\mathrm{a}}$ can be written as $\sigma_{\mathrm{a}}=\sigma_{\mathrm{a} 300 \mathrm{~K}} \exp \left(\frac{-\mathrm{E}_{\mathrm{a}, \mathrm{a}}}{\mathrm{k}_{\mathrm{B}}}\left(\frac{1}{\mathrm{~T}}-\frac{1}{300}\right)\right)$. In order to fit the experimental data below the threshold, $\sigma_{\mathrm{a} 300 \mathrm{~K}}$ is set to $3 \times 10^{-8} \Omega^{-1} \mathrm{~cm}^{-1}$, and the amorphous activation energy $\mathrm{E}_{\mathrm{a}, \mathrm{a}}$ is adjusted to $0.75 \mathrm{eV}$.

The conductivity of the crystalline silicon is related to the Fermi level position involved at large grain boundaries. $\sigma_{\mathrm{c}}$ can be written as $\sigma_{\mathrm{c}}=\sigma_{\mathrm{c} \text {,intrinsic 300K }} \exp \left(\frac{\mathrm{E}_{\mathrm{F}}-\mathrm{E}_{\mathrm{Fi}}}{\mathrm{k}_{\mathrm{B}} \mathrm{T}}\right)$ with $\sigma_{\mathrm{c}, \text { intrinsic 300K }}$ $=3 \times 10^{-6} \Omega^{-1} \mathrm{~cm}^{-1}$ at $300 \mathrm{~K}$ and $\mathrm{E}_{\mathrm{Fi}}$ is the intrinsic Fermi level close to the midgap. In order to be consistent with the value used in the physical model of the conductivity of the grain boundary ( ${ }^{1} \mathrm{~cm}^{-1}$ at $300 \mathrm{~K}$. 
$\sigma_{\mathrm{j}}$ has demonstrated a high influence on the conductivity above the percolation threshold. This is in agreement with the AFM experimental data and the large voltage drops at the large grain boundary. In order to fit the conductivity of the film over the experimental temperature range, $\sigma_{\mathrm{j}}$ must take the following form:

$$
\sigma_{j}=\sigma_{j 0} \exp \left(\frac{-E_{a, j}}{k_{B} T}\right)
$$

where $E_{a, j}=0.4 \mathrm{eV}$ is the activation energy corresponding to the transport through potential barrier when a percolation network is formed (crystalline fraction $>60 \%$ ), and $\sigma_{\mathrm{j} 0}=1.1 \times 10^{2}$ $\Omega^{-1} \mathrm{~cm}^{-1}$. The physical interpretation of the $\sigma_{\mathrm{j}}$ value requires development of a transport model through the large grain boundaries. The next section is devoted to this study.

$\sigma_{\mathrm{ac}}$ models the transport through an amorphous/crystalline heterojunction and has an influence only on the simulated conductivity curve around the percolation threshold. The effect of $\sigma_{\mathrm{ac}}$ on the simulated conductivity is shown on Fig.9 (solid triangles and closed circles). The results clearly show that the high value of $\sigma_{\mathrm{ac}}\left(\sigma_{\mathrm{ac}}=10^{-6} \Omega^{-1} . \mathrm{cm}^{-1}\right.$ on Fig. 9) induces a progressive increase in the conductivity with increasing crystalline fraction around the percolation threshold.

A low value of $\sigma_{\mathrm{ac}}\left(\sigma_{\mathrm{ac}}=1.2 \times 10^{-10} \Omega^{-1} \cdot \mathrm{cm}^{-1}\right.$, closed circles on Fig. 9) induces a slight decrease of the simulated conductivity below the percolation threshold. This behavior is in agreement with our experimental data. Below the percolation threshold, the electrical transport occurs in the amorphous silicon matrix. The few isolated crystallites do not contribute significantly to the current due to the barrier of the amorphous/crystalline silicon interface. Thus, crystallites reduce the conductive paths in the amorphous conductive medium.

\subsection{Large grain boundary model}

According to the experimental results presented in section 4, the conductivity at high crystallinity is dominated by large grain boundaries. Barrier-limited transport has been proposed to describe the charge transport through these regions whether by thermionic emission [8,21], tunneling channels [22], hopping via band tail states [16] or by a combination of these mechanisms according to the temperature range [18,23-25]. In the range of our measurement temperatures, we expect that tunnel junction model will capture the behavior of the electrical transport at large grain boundaries as depicted in Fig. 10. The density of tunnel current crossing this barrier is described by the Tsu-Esaki formula [26]: 


$$
J(V)=\frac{4 q m}{h^{3}} \int_{E_{\min }}^{E_{\max }} T_{C}(E) N(E) d E
$$

$m$ is defined as the mass of the electron; $E_{\min }$ and $E_{\max }$ are respectively the conduction band level in region 1 and region $2 . \mathrm{T}_{\mathrm{C}}(\mathrm{E})$ is the transmission coefficient which defines the transport mechanism and changes depending on E, the energy of the electron. $N(E)$ is the supply function assuming Fermi-Dirac statistics. $\mathrm{T}_{\mathrm{C}}(\mathrm{E})$ is calculated by solving the Schrödinger equation within the effective mass equation approximation. This numerical approach allows taking into account of direct and Fowler-Nordheim tunneling, and thermionic emission.

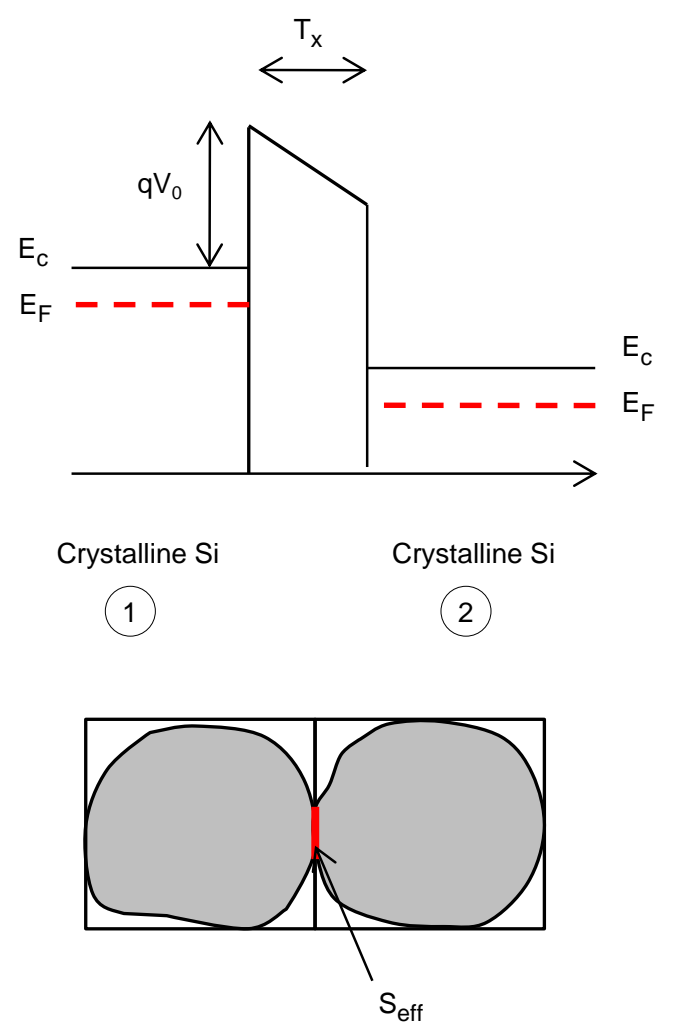

Fig. 10. Diagram of the potential barrier between two crystalline grains subjected to a potential V.

The conductivity of the barrier varies depending on the temperature, the barrier width $\left(\mathrm{T}_{\mathrm{x}}\right)$, the barrier height $\left(\mathrm{qV}_{0}\right)$, the position of Fermi level $\left(\mathrm{E}_{\mathrm{c}}-\mathrm{E}_{\mathrm{F}}\right)$, the effective contact surface between grains ( $S_{\text {eff }}$ ) and the applied voltage $V$. Based on the large number of tunnel junction distributed in the whole material, we assume that the applied voltage $\mathrm{V}$ across the junction is small, so that $\sigma_{\mathrm{j}}$ is related to the linearization of $\mathrm{J}$ with respect to the applied voltage at $\mathrm{V}=0$ and the network unit $\mathrm{u}$ : 


$$
\sigma_{\mathrm{j}}=\mathrm{u} \frac{\mathrm{dJ}}{\mathrm{dV}}
$$

Within this approximation, all dipoles involved in the equivalent circuit are linear and computation time to solve the circuit is drastically reduced.

At high crystalline fraction, the electrical transport is dominated by tunnel junctions, and experimental data exhibit activation energy of $0.4 \mathrm{eV}$. The temperature behavior of the conductivity is mainly related to $\sigma_{\mathrm{j}}$ which is modeled with the Tsu-Esaki formula. We observed that $\sigma_{\mathrm{j}}$ behaves as an Arrhenius law (Fig. 11), and the slope is related to the Fermi level position $E_{c}-E_{F}$. The Fermi level position is adjusted to fit the experimental activation energy.

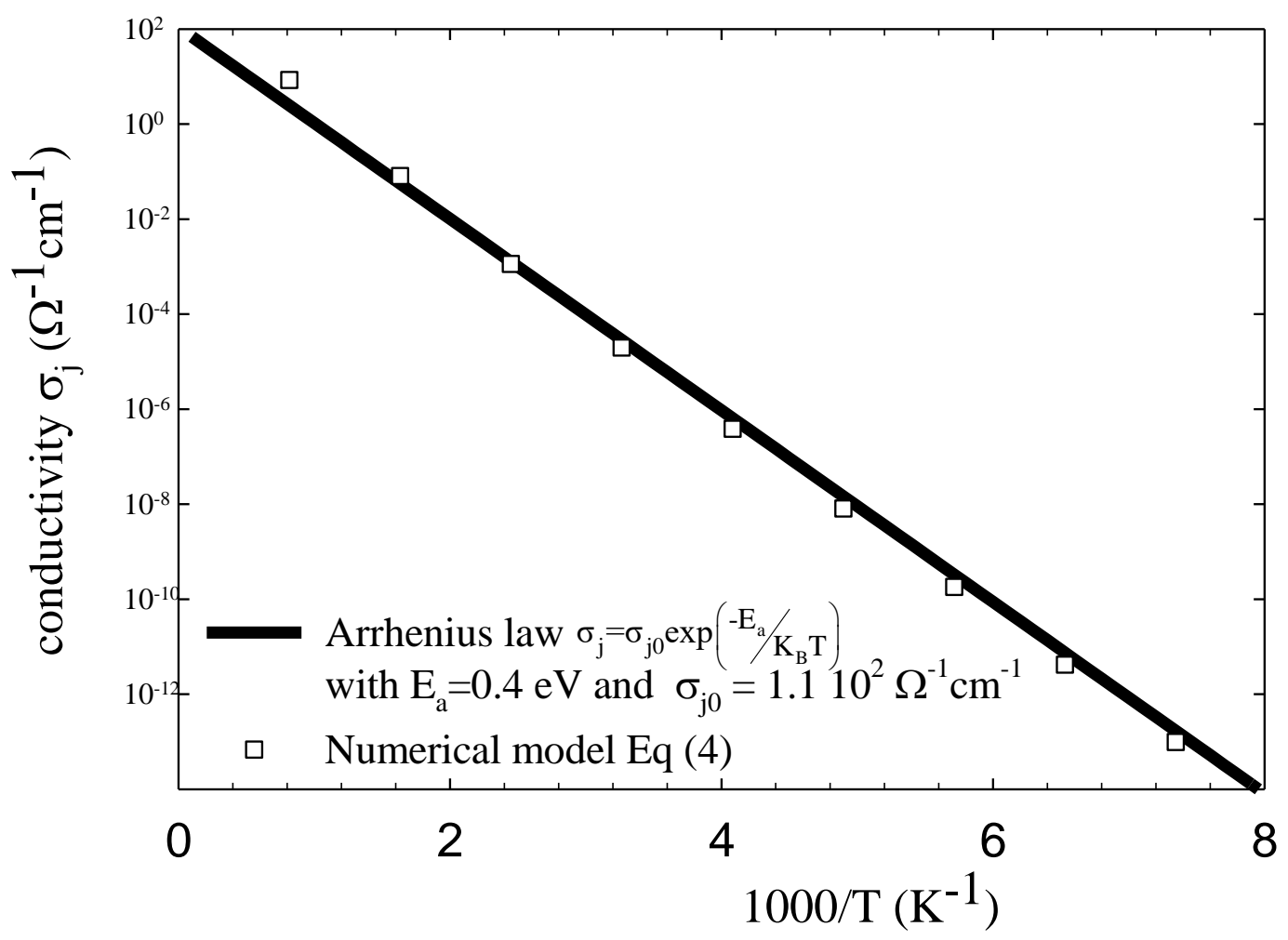

Fig. 11 : Conductivity of the grain boundary $\sigma_{j}$ between two small cubes calculated with Eq. 4 (open squares) compared to Arrhenius law (Eq. 2) (solid line). Other parameters are given in the table 2.

The other parameters are adjusted in order to fit the conductivity of high crystalline samples presented in Fig. 9. The best fit is obtained with parameters presented in Table 2. 


\begin{tabular}{llll}
\hline Barrier thickness & Barrier height & Effective contact area & Fermi level \\
\hline $\mathrm{T}_{\mathrm{x}}=0.5 \mathrm{~nm}$ & $\mathrm{qV}_{0}=0.1 \mathrm{eV}$ & $\mathrm{S}_{\mathrm{eff}}=9 \mathrm{~nm}^{2}$ & $\mathrm{E}_{\mathrm{c}}-\mathrm{E}_{\mathrm{F}}=0.373 \mathrm{eV}$ \\
\hline
\end{tabular}

Table 2 : Parameters of the potential barrier between two cubes at the grain boundaries.

The parameters in Table 2 are consistent with the published results on the characteristics of a potential barrier between two crystalline silicon grains [17]. The effective contact surface between grains is also coherent with the network unit $(3 \mathrm{~nm})$ used by the growth simulation. The Fermi level located at $0.37 \mathrm{eV}$ from the conduction band can be attributed to a nonintentional doping in the grains.

The reasonable values of the parameters strengthen the assumption of the transport dominated by the tunneling at grain boundary.

\section{Conclusion}

Electrical characterizations have been performed on $\mu \mathrm{c}-\mathrm{Si}: \mathrm{H}$ for various crystalline fractions. Percolation transport has been observed with a threshold around 55\%. The activation energy is around $0.7 \mathrm{eV}$ for low crystalline fraction and drops to $0.35 \mathrm{eV}$ above the percolation threshold.

Moreover, surface potential measurements have been carried out on the samples between two biased electrodes. For a sample of low crystalline fraction, the potential decreases linearly. In a high crystallinity sample, the potential drops at the large grain boundaries, while the potential is quite constant inside each grain. These observations led us to the conclusion that the large grain boundaries constitute a potential barrier to the current with a lower conductivity than inside the large crystalline grain. Combining these experimental results, we conclude that the electrical transport takes place in the amorphous matrix below the percolation threshold, whereas, above the percolation threshold, large crystalline grains create conductive paths whereby the conductivity is limited by the grains boundaries.

In order to investigate the electrical properties, we have developed a 3D numerical model of the $\mu \mathrm{c}-\mathrm{Si}: \mathrm{H}$. The multiscale challenge of this model has been addressed with the generation of an electrical circuit. The discretization of the material is given by a $3 \mathrm{D}$ growth model, providing a very accurate structural model.

A good agreement is obtained between the conductivity of the samples and the model. Typical values for the crystalline and amorphous silicon conductivities have been extracted. The role of the crystallites at low crystalline fraction has been investigated, and the reduction 
of the conductive paths through the amorphous matrix could explain the slight decrease in conductivity while increasing the crystalline fraction below the percolation threshold.

Special attention has been paid to the physical modeling of the tunneling at large grain boundary. The extracted parameters are in agreement with published results. This tunnel junction model provides a physical meaning to the empirical value of $\sigma_{\mathrm{j}}$.

Combining the latter with other conductivities in the large electrical circuit, the percolation behavior in respect to crystalline fraction is fully simulated. A benefit of such a model is that it captures the essential physics phenomena with a few comprehensible parameters. The Monte-Carlo nature of the growth model also obviously induces the consideration of conductivity dispersion for a given process. This prediction could be of great interest for simulation of devices using $\mu \mathrm{c}-\mathrm{Si}: \mathrm{H}$.

\section{Funding:}

This work was supported by the French "Investments for the future" program managed by the National Agency for Research under contract ANR-10-LABX-22-01-SOLSTICE

\section{References}

[1] C-H. Lee, A. Sazonov, A. Nathan, High-mobility nanocrystalline silicon thin-film transistors fabricated by plasma-enhanced chemical vapor deposition, Appl. Phys. Lett. 86 (2005) 222106.

[2] B. Yan, G. Yue, X. Xu, J. Yang and S. Guha, High efficiency amorphous and nanocrystalline silicon solar cells, Phys. Status Solidi 207 (2010) 671-677

[3] K. Shimakawa, Electronic and optical properties of hydrogenated microcrystalline silicon: review, J. Mater. Sci. Mater. Electron. 15 (2004) 63-67.

[4] A. Fejfar, A. Vetushka, V. Kalusová, O. Čertík, M. Ledinský, B. Rezek, et al., Relation of nanoscale and macroscopic properties of mixed-phase silicon thin films, Phys. Status Solidi A 207 (2010) 582-586.

[5] S.J. Konezny, M.N. Bussac, L. Zuppiroli, Charge transport mechanisms in microcrystalline silicon, Appl. Phys. Lett. 92 (2008) 12107.

[6] K. Shimakawa, Percolation-controlled electronic properties in microcrystalline silicon: effective medium approach, J. Non-Cryst. Solids 266 (2000) 223-226.

[7] Y. He, Y. Wei, G. Zheng, M. Yu, M. Liu, An exploratory study of the conduction mechanism of hydrogenated nanocrystalline silicon films, J. Appl. Phys. 82 (1997) 3408-3413.

[8] H. Overhof, M. Otte, M. Schmidtke, U. Backhausen, R. Carius, The transport mechanism in micro-crystalline silicon, J. Non-Cryst. Solids vol 227 (1998) 992-995.

[9] J. Bailat, E. Vallat-Sauvain, A. Vallat, A. Shah, Simulation of the growth dynamics of amorphous and microcrystalline silicon, J. NonCryst. Solids 338 (2004) 32-36.

[10] R. Amrani, F. Pichot, L. Chahed, Y. Cuminal, Amorphous-Nanocrystalline transition in silicon thin films obtained by argon diluted silane PECVD. Crystal Structure Theory and Applications. 1 (2012) 57-61.

[11] R. Amrani, P. Abboud, L. Chahed and Y. Cuminal. Low temperature growth of hydrogenated silicon prepared by PECVD from argon diluted silane plasma. Crystal Structure Theory and Applications. 1 (2012) 62-67.

[12] S.K. Ram, S. kumar, P. Roca i Cabarrocas, Normal and anti Meyer-Neldel rule in conductivity of highly crystallized undoped microcrystalline silicon films, J. Non-Cryst. Solids, 354 (2008) 2263-2267.

[13] J. Kočka, A. fejfar, H. Stuchlíková, J. Stuchlík, P. Fojtík, T. Mates, et al., Basic features of transport in microcrystalline silicon, Sol. Energ. Mat. Sol. Cells, 78 (2003) 493-512. 
[14] J. Kočka, Relation of defects and grain boundaries to transport and photo-transport: solved and unsolved problems in microcrystalline silicon, J. Non-cryst. solids, 358 (2012) 1946-1953.

[15] R. W. Lof, R. E. I. Schropp, The statistical shift of the chemical potential causing anomalous conductivity in hydrogenated microcrystalline silicon, J. Appl. Phys. 108 (2010) 63714.

[16] J. Kočka, H. Stuchlíková, J. Stuchlík, B. Rezek, T. Mates, V. Švrček, et al., model of transport in microcrystalline silicon, J. Non-Cryst. Solids 299 (2002) 355-359.

[17] D.Ruff, H. Mell, L. Toth, I. Sieber, W. fuhs, Charge transport in microcrystalline silicon films, J. Non-Cryst. solids, 227 (1998) 10111015.

[18] F. liu, M. Zhu, Y. Feng, Y. Han, J. Liu, S. Kasouit, et al., "Transport mechanism of microcrystalline silicon thin films, J. Non-Cryst. Solids, 299 (2002) 385-389.

[19] G. Sassine, F. Martinez, M. El Khoury, F. Pascal, A. Hoffmann, Measurements and simulation of surfactant's impact on the conductivity and the 1/f noise in percolation carbon nanotube networks, IEEE Trans. Electron Devices 59 (2012) 2803-2808.

[20] R. Amrani, Croissance et propriétés de couches minces de silicium hydrogéné déposées au voisinage de la zone de transition amorphe nanocristalline par PECVD à partir d'un plasma de silane dilué dans un gaz d'argon, doctoral thesis, University of Montpellier, Montpellier, France, 2013.

[21] K. Murata, K. Shimakawa, Y. Takai, T. Itoh, Photo-carrier transport in microcrystalline silicon films prepared by hot-wire CVD, Thin Solid Films. 516 (2008) 572-575.

[22] K. Lips, P. Kanschat, W. Fuhs, Defects and recombination in microcrystalline silicon, Sol. Energy Mater. Sol. Cells. 78 (2003) $513-$ 541.

[23] Z.A.K. Durrani, M.A. Rafiq, Electronic transport in silicon nanocrystals and nanochains,Microelectron. Eng. 86 (2009) 456-466.

[24] A. Dussan, R.H. Buitrago, Transport mechanism in lightly doped hydrogenated microcrystalline silicon thin films, J. Appl. Phys. 97 (2005) 43711.

[25] J.-H. Zhou, S.D. Baranovskii, S. Yamasaki, K. Ikuta, M. Kondo, A. Matsuda, et al., Transport properties of microcrystalline silicon at low temperatures, Semiconductors. 32 (1998) 807-811.

[26] R. Tsu, L. Esaki, Tunneling in a finite superlattice, Appl. Phys. Lett. 22 (1973) 562-564. 